\title{
Joint MIMO Radar Waveform and Receiving Filter Optimization
}

\author{
Chun-Yang Chen and P. P. Vaidyanathan \\ Dept. of Electrical Engineering, MC 136-93 \\ California Institute of Technology, Pasadena, CA 91125, USA \\ E-mail: cyc@caltech.edu, ppvnath@systems.caltech.edu
}

\begin{abstract}
The concept of MIMO (multiple-input multipleoutput) radar allows each transmitting antenna element to transmit an arbitrary waveform. This provides extra degrees of freedom compared to the traditional transmit beamforming approach. It has been shown in the recent literature that MIMO radar systems have many advantages. In this paper, we consider the joint optimization of waveforms and receiving filters in the MIMO radar when the prior information of target and clutter are available. A novel iterative algorithm is proposed to optimize the waveforms and receiving filters such that the detection performance can be maximized. The proposed algorithm guarantees that the SINR performance improves in each iteration step. The numerical results show that the proposed methods have better SINR performances than existing design methods.
\end{abstract}

Index Terms - MIMO Radar, Waveform Design, Extended Target, Clutter, Beamforming.

\section{INTRODUCTION}

MIMO radar waveform design problems have been studied in [5]-[8] and the references there in. These methods can be broken into three categories: (1) covariance matrix based design [6], (2) radar ambiguity function based design [7], and (3) prior information based design [5]. In the covariance matrix based design methods, the covariance matrix of the waveforms are considered instead of the entire waveform. Consequently, this kind of design methods affects only the spatial domain. In [6], the covariance matrix of the transmitted waveforms is designed such that the power can be transmitted to a desire range of angles. The radar ambiguity function based methods optimize the entire waveforms instead of just their covariances. Thus these design methods involve not only the spatial domain but also the range domain. In [7], the waveforms are optimized so that a sharper radar ambiguity function can be obtained. Thus the spatial and range resolution of point targets can be improved. The MIMO radar ambiguity function can be found in [10] and the references therein. In the prior information based methods also, the entire waveform is considered as in the radar ambiguity function based approaches. However, unlike the ambiguity function based methods which consider the resolutions of point targets, these methods consider the detection or estimation of extended targets. These methods require some prior information about the target and/or clutter impulse response. The prior information based methods have been also studied in the SIMO case [1]-[4]. In [3], [4], the mutual information between the received waveforms and the target impulse response has been optimized by properly designing the transmitting waveforms. This idea has been extended to the MIMO radar case in [8]. However, in [3], [8] the effect of the clutter is ignored. In [1], [2], the clutter impulse response has been considered. In these methods, the SINR

${ }^{1}$ Work supported in parts by the ONR grant N00014-08-1-0709 and the California Institute of Technology. has been maximized to improve the detection performance by properly designing the transmitting waveform. Both [1], [2] have proposed different iterative algorithms to maximize the SINR. For the MIMO radar, SINR maximization with both target and clutter information has been considered in [5]. A MIMO extension of the method in [2] and a gradient based method have been proposed in [5] to solve for the transmitted waveforms. Several suboptimal solutions have also been studied in [5].

In this paper, we also consider the waveform design problem which maximizes the SINR in the presence of clutter in the colocated MIMO radar case. As shown in [1], [2], [5], the difficulty of this problem is that the objective function, namely the SINR, is not a convex function of the transmitted waveforms. Moreover, it cannot be easily solved by Lagrange multiplier methods. In [1], [2], [5], different iterative methods have been developed. In [1], the algorithm guarantees the SINR to be nondecreasing in each iterative step. However, it has not been extended to the MIMO case because the algorithm is based on the symmetry of the SIMO radar ambiguity function which is no longer valid for the MIMO radar case. On the other hand, in [2], [5], the algorithms work for the MIMO radar case. However they do not guarantee nondecreasing SINR in each iteration step. Consequently, these algorithms cannot guarantee convergence.

In this paper, we propose a new algorithm which works in the MIMO radar case and guarantees nondecreasing SINR in each iteration step. This iterative algorithm alternatively solves the optimal transmitted waveforms and the receiving filters by fixing the other parameters. The numerical results show that it converges faster and has better SINR performances than the method in [2], [5].

\section{PROBLEM ForMULATION}

Consider a MIMO radar system with $N_{T}$ transmitting antennas and $N_{R}$ receiving antennas. A finite duration $N_{T} \times 1$ vector signal $\mathbf{f}(n)$ is converted to analog waveforms, modulated, and emitted. The waveforms are reflected back by the target and clutter. In the receiver, $N_{R}$ waveforms are received, demodulated and converted back to a discrete vector signal $\mathbf{r}(n)$. The signal model for our MIMO radar system is therefore as shown in Fig. 1. The received signal $\mathbf{r}(n)$ is processed by a receiving filter $\mathbf{H}(z)$ to further determine the existence of the target. It is well known that such a system can be represented by a discrete baseband equivalent model. As shown in Fig. 1, $\mathbf{T}(z)$ and $\mathbf{C}(z)$ represent the transfer functions of the target and clutter respectively. We assume $\mathbf{T}(z)$ is a known FIR filter. It can be represented as

$$
\mathbf{T}(z)=\sum_{n=0}^{L} \mathbf{t}(n) z^{-n},
$$




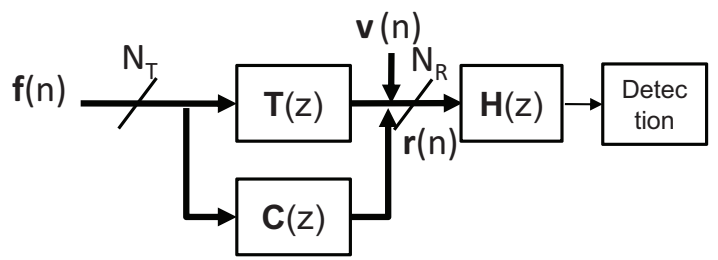

Fig. 1. Illustration of the discrete baseband equivalent signal model.

where $\mathbf{t}(n) \in \mathbb{C}^{N_{R} \times N_{T}}$ is the impulse response of the target (i.e., $\mathbf{t}_{k, l}(n)=$ impulse response from the $l$ th transmitting antenna to the $k$ th receiving antenna) and $L$ is the order of the FIR filter. The clutter transfer function can be represented as

$$
\mathbf{C}(z)=\sum_{n=-\infty}^{\infty} \mathbf{c}(n) z^{-n}
$$

where $\mathbf{c}(n) \in \mathbb{C}^{N_{R} \times N_{T}}$ is the impulse response of clutter. We assume $\operatorname{vec}(\mathbf{c}(n))$ is a vector wide-sense stationary (WSS) process with known covariance

$$
\mathbf{R}_{c}(m) \triangleq E\left[\operatorname{vec}(\mathbf{c}(n)) \operatorname{vec}(\mathbf{c}(n-m))^{\dagger}\right],
$$

where the notation $\operatorname{vec}(\mathbf{A})$ denotes a vector formed by reshaping the matrix $\mathbf{A}$. The $N_{R} \times 1$ vector process $\mathbf{v}(n)$ shown in Fig. 1 represents the noise in the receiver. We also assume the covariance

$$
\mathbf{R}_{v}(m) \triangleq E\left[\mathbf{v}(n) \mathbf{v}(n-m)^{\dagger}\right]
$$

is known. The assumption of the availability of this prior information has also been made in [1], [2], [5].

With the prior information of the target impulse response and the second order statistics of the clutter impulse response and noise, our goal is to jointly design the $N_{T} \times 1$ transmitted vector waveform $\mathbf{f}(n)$ and the $N_{R} \times 1$ receiving filter $\mathbf{H}(z)$ to maximize the detection rate. It is well-known that the optimal detection can be obtained by the log-likelihood ratio test. In this case, the detection rate is a nondecreasing function of the SINR. Therefore, our goal becomes to maximize the SINR by choosing $\mathbf{f}(n)$ and $\mathbf{H}(z)$. The single-input singleoutput (SISO) case of this problem, where $N_{R}=N_{T}=1$, has been studied by DeLong and Hofstetter in 1967 [1] and more recently by Pillai et al. [2]. Two different types of iterative methods have been proposed for solving this problem. DeLong and Hofstetter's iterative method takes advantage of the symmetry property of the cross ambiguity function. This method guarantees the SINR to be nondecreasing in each iteration step. Nevertheless, the symmetry property cannot be applied in the general MIMO case. Consequently this method cannot be generalized to the MIMO case. On the other hand, Pillai's method has been generalized to the MIMO case by Friedlander [5]. However, this method does not guarantee the SINR to be nondecreasing in each iteration step. In this paper, we propose a new iterative method for optimizing the MIMO radar transceiver. It works in the MIMO case and also guarantees the SINR to be nondecreasing in every iteration step. The received baseband waveform $\mathbf{r}(n)$ can be expressed as

$$
\mathbf{r}(n)=\sum_{m=0}^{L_{T}}(\mathbf{t}(n-m)+\mathbf{c}(n-m)) \cdot \mathbf{f}(m)+\mathbf{v}(n),
$$

where $L_{T}$ is the order of the finite duration signal $\mathbf{f}(n)$. We define

$$
\mathbf{r} \triangleq\left[\mathbf{r}(0) ; \mathbf{r}(1) ; \cdots ; \mathbf{r}\left(L_{R}\right)\right] \in \mathbb{C}^{N_{R}\left(L_{R}+1\right) \times 1},
$$

where $L_{R}$ is the order of the receiving filter $\mathbf{H}(z)$. Then the overall received signal can be expressed as

$$
\mathbf{r}=(\mathbf{T}+\mathbf{C}) \mathbf{f}+\mathbf{v},
$$

where

$$
\begin{aligned}
\mathbf{f} & \triangleq\left[\mathbf{f}(0) ; \mathbf{f}(1) ; \cdots ; \mathbf{f}\left(L_{T}\right)\right] \in \mathbb{C}^{N_{T}\left(L_{T}+1\right) \times 1}, \\
\mathbf{v} & \triangleq\left[\mathbf{v}(0) ; \mathbf{v}(1) ; \cdots ; \mathbf{v}\left(L_{R}\right)\right] \in \mathbb{C}^{N_{R}\left(L_{R}+1\right) \times 1},
\end{aligned}
$$

and $\mathbf{T}$ and $\mathbf{C}$ are the corresponding block Toeplitz matrices consisting of $\mathbf{t}(n)$ and $\mathbf{c}(n)$ respectively. The receiving filter output can be expressed as

$$
\mathbf{y}=\mathbf{h}^{\dagger} \mathbf{r}=\underbrace{\mathbf{h}^{\dagger} \mathbf{T f}}_{\text {signal }}+\underbrace{\mathbf{h}^{\dagger} \mathbf{C f}}_{\text {clutter }}+\underbrace{\mathbf{h}^{\dagger} \mathbf{v}}_{\text {noise }} .
$$

Thus the SINR at the filter output can be expressed as

$$
\rho(\mathbf{f}, \mathbf{h}) \triangleq \frac{\left|\mathbf{h}^{\dagger} \mathbf{T f}\right|^{2}}{E\left[\left|\mathbf{h}^{\dagger} \mathbf{C f}\right|^{2}\right]+E\left[\left|\mathbf{h}^{\dagger} \mathbf{v}\right|^{2}\right]} .
$$

Our goal is to maximize the SINR subject to the power constraint, that is,

$$
\max _{\mathbf{f}, \mathbf{h}} \rho(\mathbf{f}, \mathbf{h}) \text { subject to }\|\mathbf{f}\|^{2} \leq 1 .
$$

One can first observe that this problem is in general not convex because the objective function is a fourth order rational function. In general, there will be multiple local maxima in the feasible set. It is in general not easy to find the global maximum.

\section{Proposed Iterative Method}

In this section, a new iterative algorithm is introduced for solving the SINR maximization problem in (5). The technique applied here is that we first optimize the receiving filter $\mathbf{h}$ for fixed transmitted waveforms $\mathbf{f}$ and then optimize $\mathbf{f}$ for fixed receiving filter $\mathbf{h}$. It can be shown that the algorithm gives a solution which is not only a local optimum, but also the global optimum separately along the $\mathbf{f}$ dimension and the $\mathbf{h}$ dimension.

We first solve $\mathbf{h}$ in terms of $\mathbf{f}$. In this case, the optimization problem becomes

$$
\max _{\mathbf{h}} \frac{\left|\mathbf{h}^{\dagger} \mathbf{T f}\right|^{2}}{\mathbf{h}^{\dagger} \mathbf{R}_{c, f} \mathbf{h}+\mathbf{h}^{\dagger} \mathbf{R}_{v} \mathbf{h}},
$$

where $\mathbf{R}_{c, f} \triangleq E\left[\mathbf{C f f}^{\dagger} \mathbf{C}^{\dagger}\right]$ and $\mathbf{R}_{v}=E\left[\mathbf{v v}^{\dagger}\right]$, where $\mathbf{v}$ is defined in (3). Note that $\mathbf{R}_{c, f}$ can be obtained by using the clutter covariance $\mathbf{R}_{c}(m)$ in (1) and $\mathbf{R}_{v}$ can be obtained by using the noise covariance $\mathbf{R}_{v}(m)$ in (2). Follows from [9] and the solution to this problem is given by

$$
\mathbf{h}=\alpha\left(\mathbf{R}_{c, f}+\mathbf{R}_{v}\right)^{-1} \mathbf{T f} .
$$

where $\alpha$ is arbitrary nonzero scalar. 
To solve $\mathbf{f}$ in terms of $\mathbf{h}$, the following optimization problem is considered.

$$
\begin{aligned}
& \max _{\mathbf{f}} \frac{\left|\mathbf{h}^{\dagger} \mathbf{T f}\right|^{2}}{\mathbf{f}^{\dagger} \mathbf{R}_{c, h} \mathbf{f}+\mathbf{h}^{\dagger} \mathbf{R}_{v} \mathbf{h}} \\
& \text { subject to }\|\mathbf{f}\|^{2} \leq 1,
\end{aligned}
$$

where $\mathbf{R}_{c, h} \triangleq E\left[\mathbf{C}^{\dagger} \mathbf{h} \mathbf{h}^{\dagger} \mathbf{C}\right]$ and $\mathbf{R}_{v} \triangleq E\left[\mathbf{v} \mathbf{v}^{\dagger}\right]$, where $\mathbf{v}$ is defined in (3). Note that both $\mathbf{R}_{c, h}$ and $\mathbf{R}_{v}$ can be obtained by using the prior second order information defined in (1) and (2). We first look at the Lagrange multiplier method to solve this problem. The Lagrangian can be defined as

$$
L(\mathbf{f}, \lambda) \triangleq \frac{\left|\mathbf{h}^{\dagger} \mathbf{T f}\right|^{2}}{\mathbf{f}^{\dagger} \mathbf{R}_{c, h} \mathbf{f}+\mathbf{h}^{\dagger} \mathbf{R}_{v} \mathbf{h}}+\lambda\left(\mathbf{f}^{\dagger} \mathbf{f}-1\right), \quad \lambda \geq 0,
$$

where $\lambda$ is the Lagrange multiplier. Differentiating the above function with respect to $\mathrm{f}$ and letting it to zero, we obtain

$$
\frac{\mathbf{T}^{\dagger} \mathbf{f h}^{\dagger} \mathbf{T} \mathbf{f}\left(\mathbf{f}^{\dagger} \mathbf{R}_{c, h} \mathbf{f}+\mathbf{h}^{\dagger} \mathbf{R}_{v} \mathbf{h}\right)-\left|\mathbf{h}^{\dagger} \mathbf{T f}\right|^{2} \mathbf{R}_{c, h} \mathbf{f}}{\left(\mathbf{f}^{\dagger} \mathbf{R}_{c, h} \mathbf{f}+\mathbf{h}^{\dagger} \mathbf{R}_{v} \mathbf{h}\right)^{2}}+\lambda \mathbf{f}=0 .
$$

One can see that the above equation has a high order polynomial of $\mathbf{f}$ in the numerator. This makes it hard to solve in general. To overcome this difficulty, we recast the problem by using the following proposition.

Proposition 1. If $\mathbf{f}_{\star}$ solves the optimization problem

$$
\max _{f} \frac{\left|\mathbf{h}^{\dagger} \mathbf{T f}\right|^{2}}{\mathbf{f}^{\dagger} \mathbf{R}_{c, h} \mathbf{f}+\mathbf{h}^{\dagger} \mathbf{R}_{v} \mathbf{h} \cdot \mathbf{f}^{\dagger} \mathbf{f}}
$$

then $\mathbf{f}_{\star \star} \triangleq \mathbf{f}_{\star} /\left\|\mathbf{f}_{\star}\right\|$ solves $(7)$.

Proof: For any $\mathbf{f} \in \mathbb{C}^{N_{T}\left(L_{T}+1\right) \times 1}$ satisfying $\|\mathbf{f}\|^{2} \leq 1$,

$$
\begin{aligned}
\frac{\left|\mathbf{h}^{\dagger} \mathbf{T} \mathbf{f}_{\star \star}\right|^{2}}{\mathbf{f}_{\star \star}^{\dagger} \mathbf{R}_{c, h} \mathbf{f}_{\star \star}+\mathbf{h}^{\dagger} \mathbf{R}_{v} \mathbf{h}} & =\frac{\left|\mathbf{h}^{\dagger} \mathbf{T} \mathbf{f}_{\star}\right|^{2}}{\mathbf{f}_{\star}^{\dagger} \mathbf{R}_{c, h} \mathbf{f}_{\star}+\mathbf{h}^{\dagger} \mathbf{R}_{v} \mathbf{h} \cdot \mathbf{f}_{\star}^{\dagger} \mathbf{f}_{\star}} \\
\geq \frac{\left|\mathbf{h}^{\dagger} \mathbf{T f}\right|^{2}}{\mathbf{f}^{\dagger} \mathbf{R}_{c, h} \mathbf{f}+\mathbf{h}^{\dagger} \mathbf{R}_{v} \mathbf{h} \cdot \mathbf{f}^{\dagger} \mathbf{f}} & \geq \frac{\left|\mathbf{h}^{\dagger} \mathbf{T f}\right|^{2}}{\mathbf{f}^{\dagger} \mathbf{R}_{c, h} \mathbf{f}+\mathbf{h}^{\dagger} \mathbf{R}_{v} \mathbf{h}} .
\end{aligned}
$$

The first inequality is because of the definition of $\mathbf{f}_{\star}$. The second inequality is from the fact that $\|\mathbf{f}\|^{2} \leq 1$. We also have $\left\|\mathbf{f}_{\star \star}\right\|^{2}=1$. Therefore $\mathbf{f}_{\star \star}$ is a solution to (7).

The solution to the optimization problem in (8) is

$$
\mathbf{f}=\alpha\left(\mathbf{R}_{c, h}+\mathbf{h}^{\dagger} \mathbf{R}_{v} \mathbf{h} \cdot \mathbf{I}\right)^{-1} \mathbf{T}^{\dagger} \mathbf{h},
$$

where $\alpha$ is arbitrary nonzero scalar.

Now we know how to solve $\mathbf{h}$ in terms of $\mathbf{f}$ and $\mathbf{f}$ in terms of $\mathbf{h}$. We can iteratively solve the transmitted waveforms $\mathbf{f}$ and the receiving filter $\mathbf{h}$. Thus the objective function, namely SINR, will be nondecreasing in every iteration step. The algorithm is summarized as follows.

Algorithm 1. Given the target impulse response $\mathbf{T}(z)$, noise covariance $\mathbf{R}_{v}(m)$, the clutter covariance $\mathbf{R}_{c}(m)$, and an initial value of the transmitted waveforms $\mathbf{f}$, the transceiver pair $(\mathbf{f}, \mathbf{h})$ can be optimized by repeating the following steps:

$$
\begin{array}{ll}
\text { 1. } & \text { Compute } \mathbf{R}_{c, f}=E\left[\mathbf{C f f}^{\dagger} \mathbf{C}^{\dagger}\right] \\
\text { 2. } & \mathbf{h} \leftarrow\left(\mathbf{R}_{c, f}+\mathbf{R}_{v}\right)^{-1} \mathbf{T f} \\
\text { 3. } & \text { Compute } \mathbf{R}_{c, h}=E\left[\mathbf{C}^{\dagger} \mathbf{h h}^{\dagger} \mathbf{C}\right] \\
\text { 4. } & \mathbf{f} \leftarrow\left(\mathbf{R}_{c, h}+\mathbf{h}^{\dagger} \mathbf{R}_{v} \mathbf{h} \cdot \mathbf{I}\right)^{-1} \mathbf{T}^{\dagger} \mathbf{h} \\
\text { 5. } & \mathbf{f} \leftarrow \mathbf{f} /\|\mathbf{f}\| .
\end{array}
$$

We stop when the SINR improvement becomes insignificant.

\section{Numerical Results}

In this section, the SINR performances of the proposed method are compared to Pillai's method [2] and the orthogonal LFM (linear frequency modulation) waveforms. The orthogonal LFM waveforms is defined as

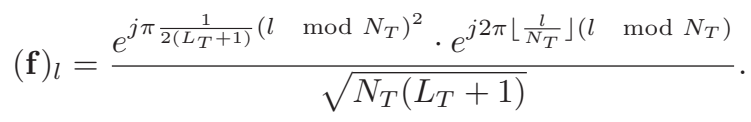

Note that LFM waveforms are designed for a different purpose, namely, obtaining sharp ambiguity function. They are good candidates for distinguish point targets and imaging. However, the LFM waveforms may not have good SINR performances in the extended target case. The matched filter bound has also been compared in the simulation; it is the optimal solution in the clutter free case, and serves as an upper bound for all the methods.

Example 1: SINR versus number of iterations.

Consider a MIMO radar system with number of transmitting antennas $N_{T}=4$ and number of receiving antennas $N_{R}=4$. Let the target impulse response be given by

$$
(\mathbf{t}(n))_{k, l}=\left\{\begin{array}{ll}
1, & n=0,1, \cdots, 20 \\
0, & \text { otherwise }
\end{array} .\right.
$$

The clutter impulse response is modelled as an AR (autoregressive) process with covariance

$$
\mathbf{R}_{c}(n)=\mathbf{B} \mathbf{A}^{|n|} \mathbf{B}^{\dagger},
$$

where the matrix $\mathbf{A}$ is a positive semidefinite matrix with spectral radius less than unity. Both the parameters $\mathbf{A}$ and $\mathbf{B}$ are randomly generated. The noise $\mathbf{v}(n)$ is modelled as white noise with unity variance. Fig. 2 shows the SINR performances defined in (4) as a function of the number of iterations. Note that LFM waveform is fixed, so its SINR

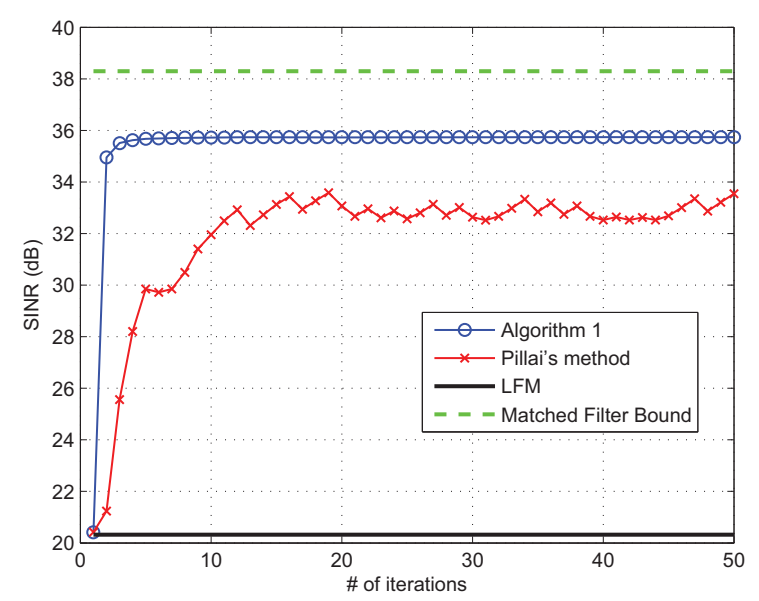

Fig. 2. Example 1: Comparison of the SINR versus number of iterations.

is not a function of the number of iterations. The initial waveform used in Algorithm 1 and Pillai's method are 

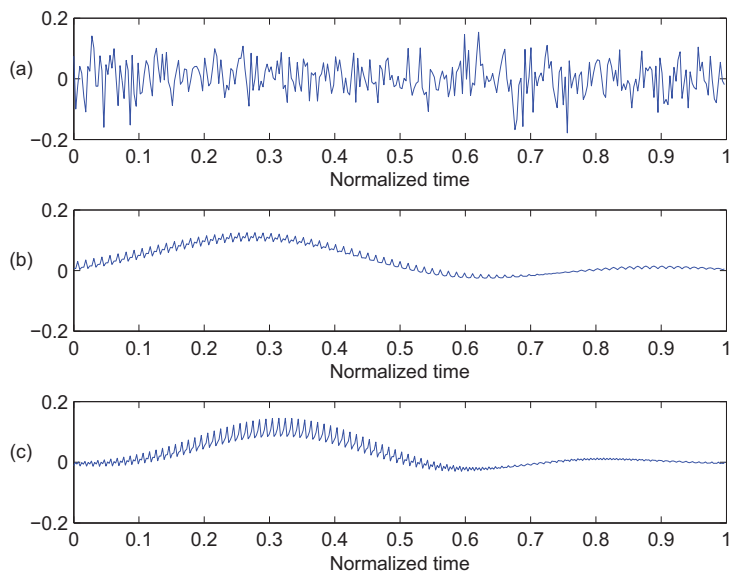

Fig. 3. Example 1: (a) the initial waveform, (b) the optimized waveform $\mathbf{f}$, and (c) the optimized receiving filter $\mathbf{h}$.

identical. It is shown in Fig. 3 (a). One can observe that Algorithm 1 has a better performance than other methods. Algorithm 1 also converges very fast. It converges in about six iterations in this example. Moreover, in Algorithm 1, the SINR is a nondecreasing function of the number of iterations. The resulting waveform $\mathbf{f}$ and receiving filter $\mathbf{h}$ are shown in Fig. 3 (b) and (c).

Example 2: SINR versus CNR.

In this example, the SINR performances are compared for different values of CNR (clutter-to-noise ratio). Consider a MIMO radar with number of transmitting antennas $N_{T}=2$ and number of receiving antennas $N_{R}=2$. The order of the target impulse response $\mathbf{T}(z)$ is 20 . The coefficients $\left\{(\mathbf{t}(n))_{k, l}\right\}$ are generated as i.i.d. (independent and identically distributed) circular complex Gaussian random variables with unity variance. The covariance of the clutter impulse response $\mathbf{R}_{c}(n)$ is generated by using

$$
\mathbf{R}_{c}(n)=\mathbf{U}_{c}(n) * \mathbf{U}_{c}(-n)^{\dagger},
$$

where the notation $*$ denotes convolution, $\mathbf{U}_{c}(n)$ is a $4 \times 4$ matrix sequence with length 31 and the coefficients $\left\{(\mathbf{U}(n))_{k, l}\right\}$ are i.i.d. circular complex Gaussian random variables. The noise $\mathbf{v}(n)$ is a white process with unity variance. The initial waveforms used in the algorithms are randomly chosen. The simulation is performed by averaging among 1000 different target, clutter and noise realizations. Fig. 4 shows the comparison of the SINR defined in (4) under different CNR. One can see that Algorithm 1 has the best SINR performances among all the methods under all CNR. Both Algorithm 1 and Pillai's method have much better performances than the LFM waveforms. This shows that utilizing the prior information in the transmitter is very crucial for the SINR performance.

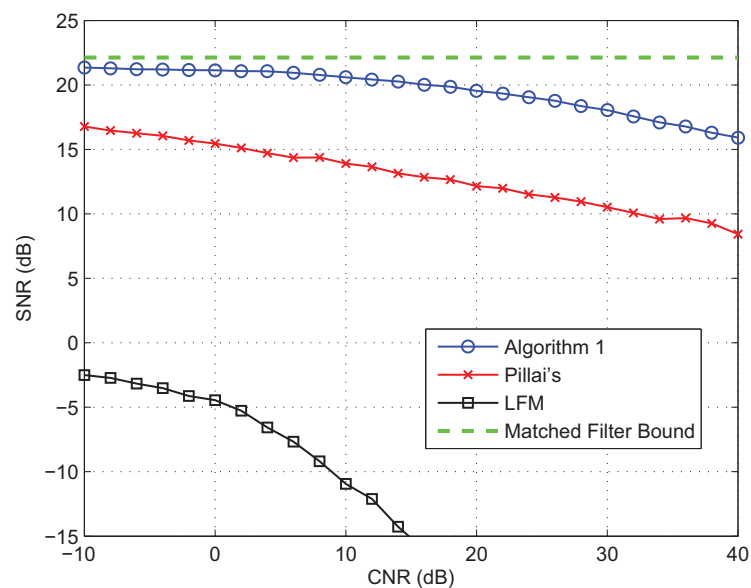

Fig. 4. Example 2: Comparison of the SINR versus CNR.

\section{CONCLUSIONS}

In this paper, we have proposed an iterative algorithm for jointly designing the transmitted waveforms and the receiving filters to maximize the SINR in the MIMO radar. This iterative algorithm alternatively solves the optimal transmitted waveforms and the receiving filters by fixing the other parameters. It can be shown that this algorithm finds a local maximum which is also a global maximum along the dimension of the transmitted waveforms and the dimension of the receiving filter separately. The numerical results show that the proposed iterative algorithm converges faster and also has better SINR performances than previously reported algorithms.

\section{REFERENCES}

[1] D. DeLong and E. Hofstetter, "On the design of optimum radar waveforms for clutter rejection," IEEE Trans. on Information Theory, Volume 13, Issue 3, pp. 454-463, July 1967.

[2] S. U. Pillai, H. S. Oh, D. C. Youla, and J. R. Guerci, "Optimal transmitreceiver design in the presence of signal-dependent interference and channel noise," IEEE Trans. on Information Theory, Volume 46, Issue 2, pp. 577-584, March 2003.

[3] M. R. Bell, "Information Theory and Radar Waveform Design," IEEE Trans. on Information Theory, Volume 39, Issue 5, pp. 1578-1597, Sept. 1993.

[4] A. Leshem, O. Naparstek, and A. Nehorai, "Information Theoretic Adaptive Radar Waveform Design for Multiple Extended Targets," IEEE Journal of Selected Topics in Signal Processing, Volume 1, Issue 1, pp. 42-55, June 2007.

[5] B. Friedlander, "Waveform Design for MIMO Radars," IEEE Transactions on Aerospace and Electronic Systems, Volume 43, Issue 3, pp 1227-1238, July 2007.

[6] P. Stoica, J. Li, and Y. Xie,"On Probing Signal Design For MIMO Radar," IEEE Trans. on Signal Processing, Volume 55, Issue 8, pp. 4151-4161, Aug. 2007

[7] C. Y. Chen and P. P. Vaidyanathan, "MIMO Radar Ambiguity Properties and Optimization Using Frequency-Hopping Waveforms," IEEE Trans. on Signal Processing, accepted for publication.

[8] Y. Yang and R. S. Blum, "MIMO Radar Waveform Design Based on Mutual Information and Minimum Mean-Square Error Estimation," IEEE Trans. on Aerospace and Electronic Systems, Volume 43, Issue 1, pp. 330343, Jan. 2007.

[9] J. Capon, "High-resolution frequency-wavenumber spectrum analysis," Proc. IEEE, Volume 57, Issue 8, pp. 1408-1418, Aug. 1969.

[10] Y. I. Abramovich and G. J. Frazer, "Bounds on the Volume and Height Distributions for the MIMO Radar Ambiguity Function," IEEE Signal Processing Letters, Volume 15, pp. 505-508, 